\title{
Application of the Kusuoka approximation to pricing barrier options
}

\author{
Shigeo Kusuoka \\ Professor emeritus at the University of Tokyo \\ Mariko Ninomiya \\ The Japanese Red Cross College of Nursing, 4-1-3 Hiroo, Shibuya-ku, Tokyo 150-0012 Japan \\ E-mail: mariko.nnmy@gmail.com \\ Syoiti Ninomiya \\ Center for Research in Advanced Financial Technology, Tokyo Institute of Technology, \\ 2-12-1 Ookayama, Meguro-ku, Tokyo 152-8552 Japan \\ E-mail: ninomiya.s.ab@m.titech.ac.jp
}

\begin{abstract}
The main theme of this research is numerical verification of applicability of a higher-order approximation to pricing barrier options, which is a both mathematically and practically important path-dependent type problem in mathematical finance. The authors successfully extend two types of algorithms called NV and NN. Both algorithms are based on a higher-order approximation scheme called Kusuoka approximation and have been shown to attain second-order approximation of stochastic differential equations as long as applied to European-type problems. In extending the algorithms, the authors apply a function representing the probability of hitting a boundary. In the numerical experiments, these two algorithms are compared with the Euler-Maruyama scheme which is one of the most popular first-order approximation schemes. As a result, it is demonstrated that the speed of calculation of these two algorithms could be much higher than that of the Euler-Maruyama scheme extended by the same way. It is concluded that one of the keys to improvement of the results is the construction of the function for calculation of the probability of hitting a boundary.
\end{abstract}

\section{Introduction}

The weak approximation is a numerical calculation of $E[f(X(T, x))]$ for a function $f$ and a diffusion process defined by a stochastic differential equation (SDE) (2). This problems has a great deal in various areas including mathematical finance. In $[7,8,9,10,12,13]$, theory and notion of higher-order approximation are introduced as well as its applicability. We call this scheme Kusuoka approximation in this paper. This scheme has been recently developed such as in $[2,3,5,16]$. Two types of algorithms for implementation of the higherorder approximation are successfully constructed in $[15$,
11] which are called NV and NN algorithms in this paper, respectively. Both algorithms attain second-order approximation for $E[f(X(T, x))]$. Higher-order approximation attains higher accuracy with the less number of partitions than first-order approximation. This fact is a great advantage in considering quasi-Monte Carlo method because the effectiveness of quasi-Monte Carlo method heavily depends on the number of dimensions of integration as discussed in Remarks 6.1 and 6.2 in [11].

Other than $E[f(X(T, x))]$, path-dependent problems represented by calculation of $E\left[h\left(\{X(s, x)\}_{0<s<T}\right)\right]$ for a functional $h:\left([0, T] \rightarrow \mathbb{R}^{N}\right) \rightarrow \mathbb{R}$ also frequently appear in mathematical finance. In particular, calculation of prices of the barrier options corresponding to

$$
E\left[g(X(T, x)), \min _{0 \leq t \leq T} X^{1}(t, x)>0\right]
$$

where $X^{1}(t, x)$ denotes the first coordinate of $X(t, x)$ and $g:[0, T] \times \mathbb{R}^{N} \rightarrow \mathbb{R}$. Here

$$
X^{j}(t, x)=x_{j}+\sum_{i=0}^{d} \int_{0}^{t} V_{i}^{j}(X(s, x)) \circ d B^{i}(s)
$$

for $j=1, \ldots, N$ where $\circ \mathrm{d} B^{i}(s)$ denotes the Stratonovich integral with respect to $B^{i}(s), B^{0}(t)=t$, $\left(B^{1}(t), \ldots, B^{d}(t)\right)$ is a $d$-dimensional standard Brownian motion, and $V_{i}=\left(X_{i}^{1}, \ldots, X_{i}^{N}\right) \in C_{b}^{\infty}\left(\mathbb{R}^{N} ; \mathbb{R}^{N}\right)$ denoting the set of $\mathbb{R}^{N}$-valued infinitely differentiable functions defined in $\mathbb{R}^{N}$ whose derivatives are all bounded.

Our aim here is to extend the NV and the NN algorithms so that they can calculate (1). We separate this problem into two parts. One corresponds to the approximation of $g(X(T, x))$ and the other the boundary condition, that is the probability at which $X(t, x)$ hits the barrier. For approximation of $g(X(T, x))$, the NV or the NN algorithm are directly applicable. For the boundary condition, we introduce the notion of killing functions which represent probability of $X(t, x) \leq 0$ for $t \in[0, T]$. Two types of killing functions are considered in this research. One is called a naive killing 
function which simply takes 1 if $X(t, x) \leq 0$ and 0 otherwise. The other killing function, called standard killing function, takes it into account the situation where $X(t, x)>0$ and $X(s, x) \leq 0$ for some $s \in(t-\Delta t, t)$. Precise definitions of these functions are given in Section 3 .

We remark that this problem is thoroughly dicussed in [4].

\section{Problems and settings}

Definitions and notations here follow the ones given in $[11]$.

Let $(\Omega, \mathcal{F}, P)$ be a probability space and $\left(B^{1}(t), \ldots, B^{d}(t)\right)$ a $d$-dimensional standard Brownian Motion. Our interest is in approximation of (1) where $g \in C_{b}^{\infty}\left(\mathbb{R}^{N} ; \mathbb{R}\right)$ and $X(t, x)$ is a solution to the Stratonovich stochastic integral equation (2) where each $V_{i} \in C_{b}^{\infty}\left(\mathbb{R}^{N} ; \mathbb{R}^{N}\right), i \in\{0,1, \ldots, d\}$, is regarded as a vector field in the following way : for $f \in C_{b}^{\infty}\left(\mathbb{R}^{N} ; \mathbb{R}\right), V_{i} f(x)=\sum_{j=1}^{N} V_{i}^{j}(x) \frac{\partial f}{\partial x_{j}}(x)$. We let $T=1$ in this paper.

\subsection{Order of approximation}

Our interest is in relation between the number of partitions and the accuracy attained by each approximation scheme.

Definition 2.1 For approximation $\left\{X_{t_{i}}^{(\operatorname{ord} p), n}\right\}_{i=0, \ldots, n}$ of $\{X(t, x)\}_{0 \leq t \leq 1} \quad$ with respect to a partition $\left(0=t_{0}<t_{1}<\cdots<t_{n}=1\right)$ of $[0,1]$, $\left|E\left[f\left(X_{1}^{(\operatorname{ord} p), n}\right)\right]-E[f(X(1, x))]\right| \quad$ is called discretization error. The number $n$ of subitervals in a partition is called size of it. If

$$
E\left[f\left(X_{1}^{(\operatorname{ord} p), n}\right)\right]=E[f(X(1, x))]+O\left(n^{-p}\right),
$$

then this approximation is said to be of order $p$.

It is shown that $p=1$ for the Euler-Maruyama(EM) scheme in $[1,6,17]$ for $C^{4}$-function $f$. Besides, $p=2$ for the NV and the NN algorithms if $f$ is Lipschitz continuous as mentioned in Introduction and shown in $[7,11,15]$.

Remark 2.1 The order of approximation is often defined with respect to the width of partition. Besides, Definition 2.1 has the following advantages:

- The total amount of calculation is determined not by the width but by the size of the partition.

- The definition above is applicable to the variable step-wise algorithms.

\subsection{Higher-order weak approximation schemes for path-independent problems}

In this paper, we take three candidates of algorithms for approximation of $g(X(1, x))$. One is the EM scheme which is one of the most popular first-order weak approximation schemes. The other two are the NV and the NN algorithms implementing the Kusuoka approximation of order 2 .

In this subsection, we introduce these three algorithms. In partitioning $[0, T]$, we take the following procedure: Let $\left(0=t_{0}^{(n)}<t_{1}^{(n)}<, \ldots,<t_{n}^{(n)}=1\right)$ be a partitioning of the interval $[0,1]$ defined by $t_{k}^{(n)}=k^{\gamma} / n^{\gamma}$, where $n \in \mathbb{N}$ and $\gamma>0$. We let $s_{k}=t_{k}^{(n)}-t_{k-1}^{(n)}$ in the following. Hence, if $\gamma \neq 1.0$, then $s_{1}, \ldots, s_{n}$ differ.

The following random variables are to be constructed in the EM scheme:

$$
\begin{aligned}
X_{0}^{(\mathrm{EM}), n}= & x \\
X_{t_{k+1}^{(n)}, n}^{(\mathrm{EM}), n}= & X_{t_{k}^{(n)}}^{(\mathrm{EM}), n}+s_{k+1} \tilde{V}_{0}\left(X_{t_{k}^{(n)}}^{(\mathrm{EM}), n}\right) \\
& +\sqrt{s_{k+1}} \sum_{i=1}^{d} V_{i}\left(X_{\left.t_{k}^{((\mathrm{)})}\right), n}^{(\mathrm{E})}\right) Z_{k+1}^{i}
\end{aligned}
$$

where $\tilde{V}_{0}$ denotes the conversion of $V_{0}$ to a drift in an Ito-form SDE. $\left\{Z_{k}^{i}\right\}_{k \in\{1, \ldots, n\}}^{i \in\{1, \ldots, d\}}$ are $n \times d$ independent random variables distributed as $N(0,1)$.

Notation 2.1 $\exp (V) x$ denotes the solution at time 1 of the ordinary differential equation (ODE)

$$
\frac{\mathrm{d} z_{t}}{\mathrm{~d} t}=V\left(z_{t}\right), \quad z_{0}=x .
$$

The NV algorithm is represented by the following family $\left\{X_{t_{i}^{(n)}}^{(\mathrm{NV}), n}\right\}_{i=0}^{n}$ of random variables :

$$
\begin{aligned}
& X_{0}^{(\mathrm{NV}), n}:=x \\
& X_{t_{k+1}^{(n)}(\mathrm{NV}), n}:= \\
& \left\{\begin{array}{c}
\exp \left(\frac{s_{k+1} V_{0}}{2}\right) \exp \left(Z_{k}^{1} U_{k}^{1}\right) \exp \left(Z_{k}^{2} U_{k}^{2}\right) \cdots \\
\cdots \exp \left(Z_{k}^{d} U_{k}^{d}\right) \exp \left(\frac{s_{k+1} V_{0}}{2}\right) X_{t_{k}^{(n)}}^{(\mathrm{NV}), n} \\
\text { if } \Lambda_{k}=+1 \\
\exp \left(\frac{s_{k+1} V_{0}}{2}\right) \exp \left(Z_{k}^{d} U_{k}^{d}\right) \exp \left(Z_{k}^{d-1} U_{k}^{d-1}\right) \cdots \\
\cdots \exp \left(Z_{k}^{1} U_{k}^{1}\right) \exp \left(\frac{s_{k+1} V_{0}}{2}\right) X_{\left.t_{k}^{(n)}\right), n}^{(\mathrm{NV})} \\
\text { if } \Lambda_{k}=-1,
\end{array}\right.
\end{aligned}
$$

where $U_{k}^{i}=\sqrt{s_{k+1}} V_{i}$ and $\left(\Lambda_{i}, Z_{i}\right)_{i \in\{0, \ldots, n-1\}}$ are $n$ independent random variables such that each $\Lambda_{i}$ is a Bernoulli random variable with $P\left(\Lambda_{i}= \pm 1\right)=1 / 2$ independent of $Z_{i}$ which is a standard $d$-dimensional normal random variable.

The NN algorithm is a parametrized family of algorithms. In this paper, the parameter $u$ in [11] is set 
equal to $3 / 4$. Then the algorithm is defined by the following family $\left\{X_{t_{i}^{(n)}}^{(\mathrm{NN}), n}\right\}_{i=0}^{n}$ of random variables :

$$
\begin{aligned}
& X_{0}^{(\mathrm{NN}), n}:=x \\
& X_{t_{k+1}^{(n)}(\mathrm{NN}), n}^{(n)}=\exp \left(\frac{s_{k+1}}{2} V_{0}+\sum_{i=1}^{d} \sqrt{s_{k+1}} S_{1, k}^{i} V_{i}\right) \\
& \exp \left(\frac{s_{k+1}}{2} V_{0}+\sum_{i=1}^{d} \sqrt{s_{k+1}} S_{2, k}^{i} V_{i}\right) X_{t_{k}^{(n)}}^{(\mathrm{NN}), n}
\end{aligned}
$$

where $\left(S_{j, k}^{i}\right)_{\substack{i \in\{1, \ldots, d\}, j \in\{1,2\} \\ k \in\{0, \ldots, n-1\}}}$ are defined by

$$
\left(\begin{array}{c}
S_{1, k}^{i} \\
S_{2, k}^{i}
\end{array}\right)=\left(\begin{array}{cc}
1 / 2 & 1 / \sqrt{2} \\
1 / 2 & -1 / \sqrt{2}
\end{array}\right)\left(\begin{array}{c}
\eta_{1, k}^{i} \\
\eta_{2, k}^{i}
\end{array}\right)
$$

and $2 \times n \times d$ independent standard normal random variables $\left\{\eta_{j, k}^{i}\right\}_{j \in\{1,2\}, k \in\{0,1, \ldots, n\}}^{i \in\{1, \ldots, d\}}$.

Remark 2.2 We need to solve some ODEs in both the NV and the NN algorithms. From Theorem 1.3, Corollary 1.4, Theorems $\mathbf{4 . 1 4}$ and $\mathbf{4 . 1 5}$ in [11], if we use fifth-or higher-order numerical methods to solve these ODEs, the NN and the $N V$ algorithms remain of order two.

\section{Operator for approximation}

\section{Notation 3.1 Let}

$$
\left(P_{t} g\right)(x)=E\left[g(X(t, x)), \min _{0 \leq s \leq t} X(s, x)>0\right] .
$$

For simplification of notation, we let $F:(0, T] \times$

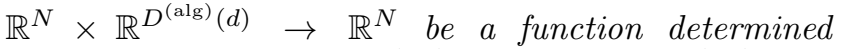
by each algorithm as $X_{0}^{(\mathrm{alg}), n}=x$ and $X_{t_{k}^{(n)}}^{(\mathrm{alg}), n}=$ $F\left(t_{k}^{(n)}, X_{t_{k-1}^{(n)}}^{(\operatorname{alg}), n}, S\left(t_{k}^{(n)}\right)\right)$, where $D^{(\operatorname{alg})}(d)$ is the number of random variables included in one-step calculation of each algorithm :

$$
D^{(\operatorname{alg})}(d)=\left\{\begin{array}{lll}
d & \text { if } & \text { alg }=\mathrm{EM} \\
d+1 & \text { if } & \text { alg }=\mathrm{NV} \\
2 d & \text { if } & \text { alg }=\mathrm{NN}
\end{array}\right.
$$

and $S\left(t_{k}^{(n)}\right)$ denotes a set of $D^{(\mathrm{alg})}(d)$ random numbers needed to obtain $X_{t_{k}^{(n)}}^{(\mathrm{alg}), n}$ from $X_{t_{k-1}^{(n)}}^{(\mathrm{alg}), n}$.

A killing function is constructed for representation of the probability density of $\{X(s, x)\}_{0 \leq s \leq t}$ hitting the boundary.

Definition 3.1 Let $k_{n}$ and $k_{s}$ be measureble functions defined on $(0,1] \times[0, \infty) \times \mathbb{R}$ as

$$
k_{n}(s, x, y)= \begin{cases}0, & \text { if } y>0 \\ 1, & \text { if } y \leq 0\end{cases}
$$

and

$$
k_{s}(s, x, y)= \begin{cases}\exp (-2 x y / s), & \text { if } y>0 \\ 1, & \text { if } y \leq 0\end{cases}
$$

We call the former naive killing function and the latter standard killing function.

Then we approximate $\left(P_{s} g\right)(x)$ by a sub-Markov operator $\left(Q_{(s)} g\right)(x)$ defined by

$$
\begin{aligned}
& \left(Q_{(s)} g\right)(x):= \\
& E[g(F(s, x, S(s)))(1-k(s, x, F(s, x, S(s))))]
\end{aligned}
$$

where $k$ is $k_{n}$ or $k_{s}$. Then $P_{T} g$ is approximated by

$$
Q_{\left(s_{n}\right)} Q_{\left(s_{n-1}\right)} \cdots Q_{\left(s_{1}\right)} g .
$$

\section{Numerical experiments}

Our focus here is on a one-dimensional SDE having the form

$$
X(t, x)=x+\int_{0}^{t} V_{0}(X(s, x)) \mathrm{d} s+\int_{0}^{t} \circ \mathrm{d} B(s) .
$$

\subsection{Drifts}

We consider (10) with the following drifts:

drift-1 $V_{0}(X(s, x))=\cos (2 \pi X(s, x))+3 / 2$

drift-2 $V_{0}(X(s, x))=\cos (10 \pi X(s, x))+3 / 2$

drift-3 $V_{0}(X(s, x))=\sqrt{(X(s, x)-1)^{2}+1}$

Remark 4.1 Singularities are avoided by adding $3 / 2$ in drift-1,2.

\subsection{Payoff functions}

The payoff functions considered in this research are a digital and a call options as defined below : for $K>0$,

digital

$$
g(y)= \begin{cases}1 & \text { if } K<y \\ 0 & \text { otherwise }\end{cases}
$$

call

$$
g(y)=\max \{0, K-y\} .
$$

4.3 Calculation with each scheme for each model

The calculations are done under the following settings:

- $\gamma=1.0$, that is $s_{k}=1 / n$ for all $k \in\{1,2, \ldots, n\}$

- The number of sample points : $4 \times 10^{8}$ 
- The size $n$ of partitions :

for EM scheme

$64,128,256,512,(1024,1536)$

for the $\mathrm{NV}$ and the $\mathrm{NN}$ algorithms

$4,8,12,16,20,32,48,64,96,128,(256,512)$

- All sample points are constructed by quasi-Monte Carlo method with a $\left(D^{(\text {alg })}(d) \times n\right)$-dimensional generalized Sobol sequence [14].

- Killing functions : both naive and standard killing functions are applied to be compared.

\subsection{True value and parameters for each model}

The true value for each combination of drifts and payoff functions is calculated by the NN algorithm with 1024 partitions, $1.2 \times 10^{8}$ sample points, and the standard killing function.

The parameters for each model are set as follows:

1. drift-1: Initial value $x=1.0$ and strike price $K=$ 1.5.

2. drift-2: Initial value $x=0.5,0.75,1.0$ and strike price $K=1.25$.

3. drift-3: Initial value $x=1.0$ and strike price $K=$ 1.25 .

We remark that all random variables are constructed by quasi-Monte Carlo method with low-discrepancy sequence.

\section{$5 \quad$ Results}

There are a number of combinations of models, initial values, payoff functions, and killing functions. The following correspondence table might be helpful to see each situation and to find the graph showing the corresponding result.

\begin{tabular}{|c||c||c|c|c|c|}
\hline \multicolumn{1}{|c||}{ drift } & \multirow{2}{*}{$\mathrm{x}$} & \multicolumn{2}{c|}{$g:$ digital } & \multicolumn{2}{c|}{$g:$ call } \\
\cline { 3 - 6 } & & $k_{n}$ & $k_{s}$ & $k_{n}$ & $k_{s}$ \\
\hline \hline 1 & 1.0 & F.5.1 & F.5.2 & F.5.3 & F.5.4 \\
\hline 2 & 1.0 & F.5.5 & F.5.6 & F.5.7 & F.5.8 \\
\cline { 2 - 6 } & 0.75 & F.5.9 & F.5.10 & F.5.11 & F.5.12 \\
\cline { 2 - 6 } & 0.5 & F.5.13 & F.5.14 & F.5.15 & F.5.16 \\
\hline 3 & 1.0 & F.5.17 & F.5.18 & F.5.19 & F.5.20 \\
\hline
\end{tabular}

The true value for each situation is shown in the following table:

\begin{tabular}{|c||c||c|c|}
\hline drift & $\mathrm{x}$ & $g:$ digital & $g:$ call \\
\hline \hline 1 & 1.0 & $8.2067927083 \mathrm{e}-01$ & $1.0606819565 \mathrm{e}+00$ \\
\hline 2 & 1.0 & $8.8313365083 \mathrm{e}-01$ & $1.2929945495 \mathrm{e}+00$ \\
\hline & 0.75 & $8.0809065667 \mathrm{e}-01$ & $1.0597288077 \mathrm{e}+00$ \\
\hline & 0.5 & $6.8119944000 \mathrm{e}-01$ & $8.1214957037 \mathrm{e}-01$ \\
\hline 3 & 1.0 & $7.8225822000 \mathrm{e}-01$ & $1.2218016886 \mathrm{e}+00$ \\
\hline
\end{tabular}

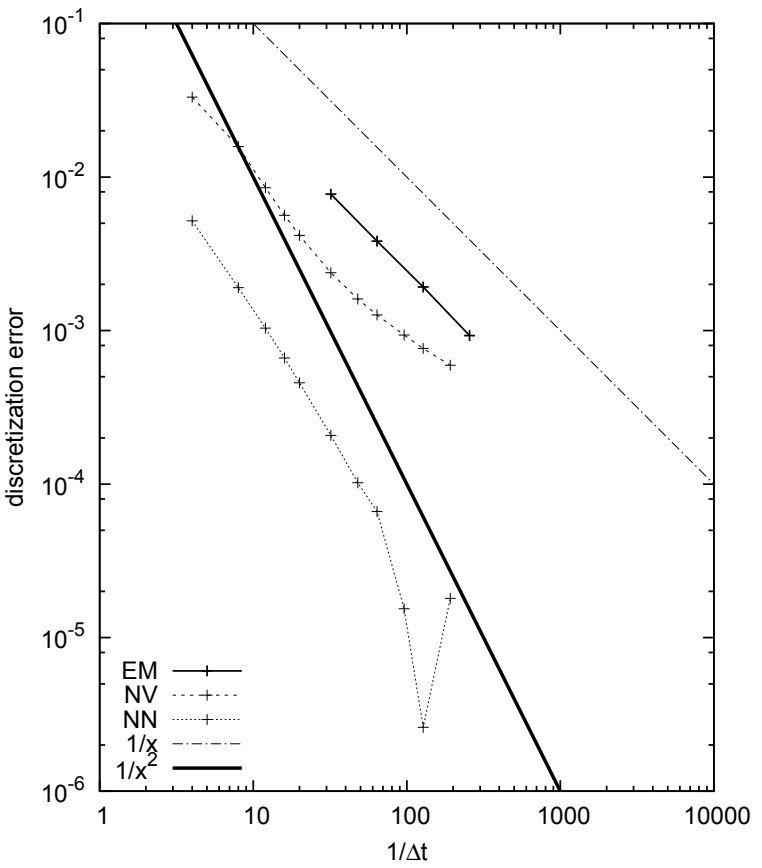

F. 5.2: (drift-1) $g:$ digital, $\quad k=k_{s}$ 


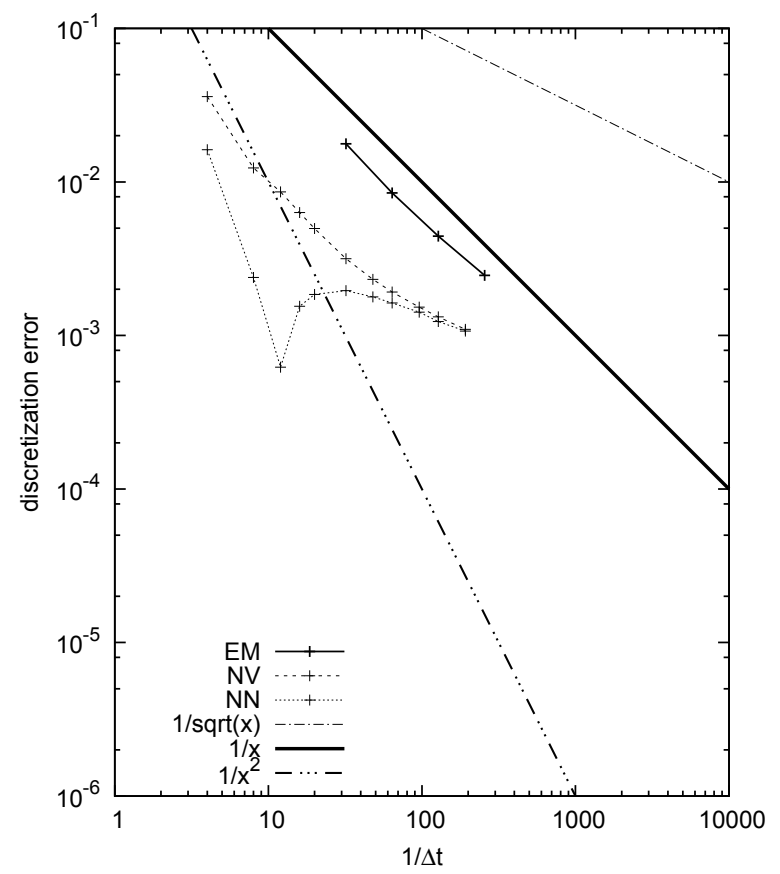

F. 5.3: (drift-1) $g:$ call, $\quad k=k_{n}$

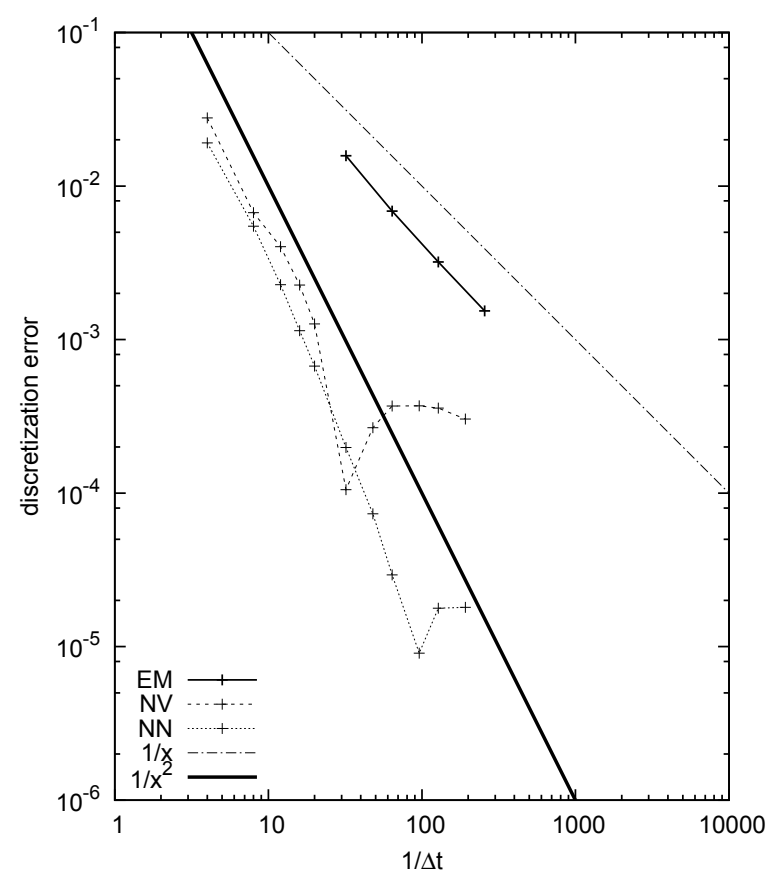

F. 5.4: (drift-1) $g:$ call, $k=k_{s}$

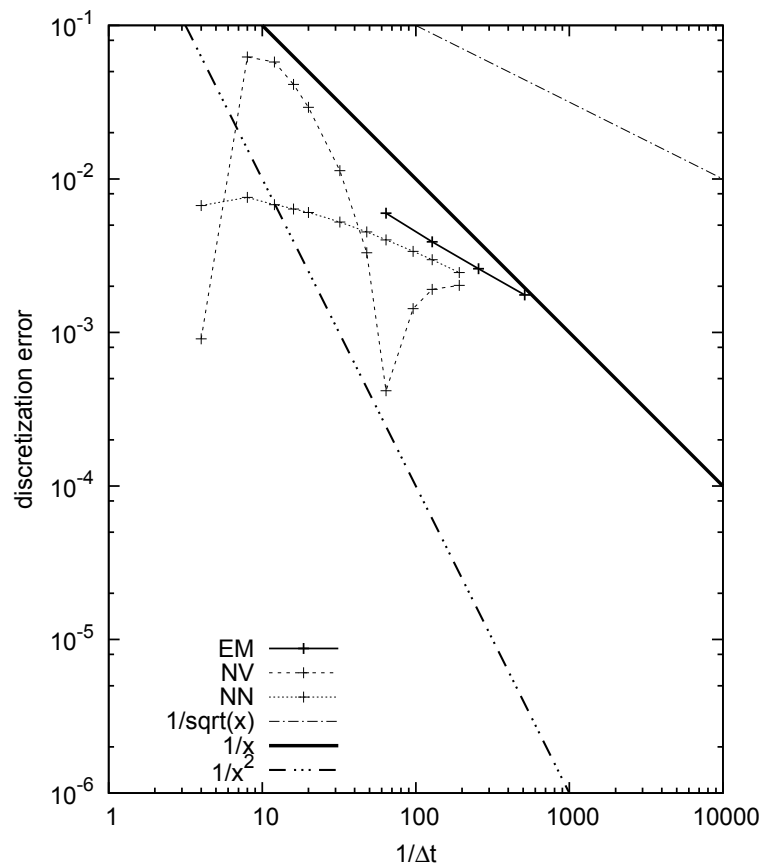

F. 5.5: (drift-2) $x=1.0, g:$ digital, $k=k_{n}$

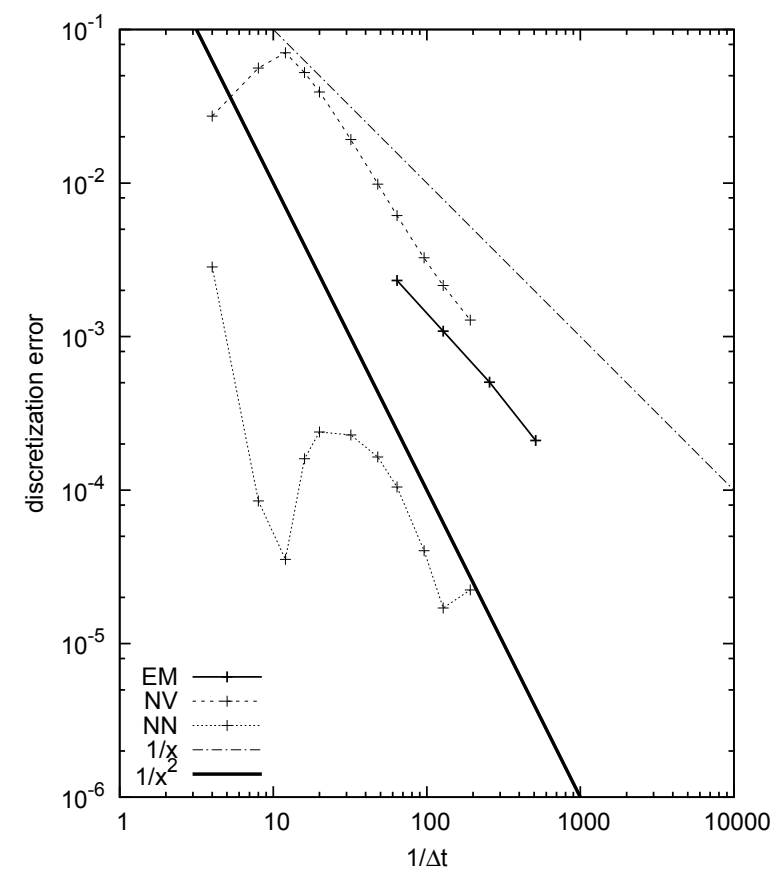

F. 5.6: (drift-2) $x=1.0, g:$ digital, $k=k_{s}$ 


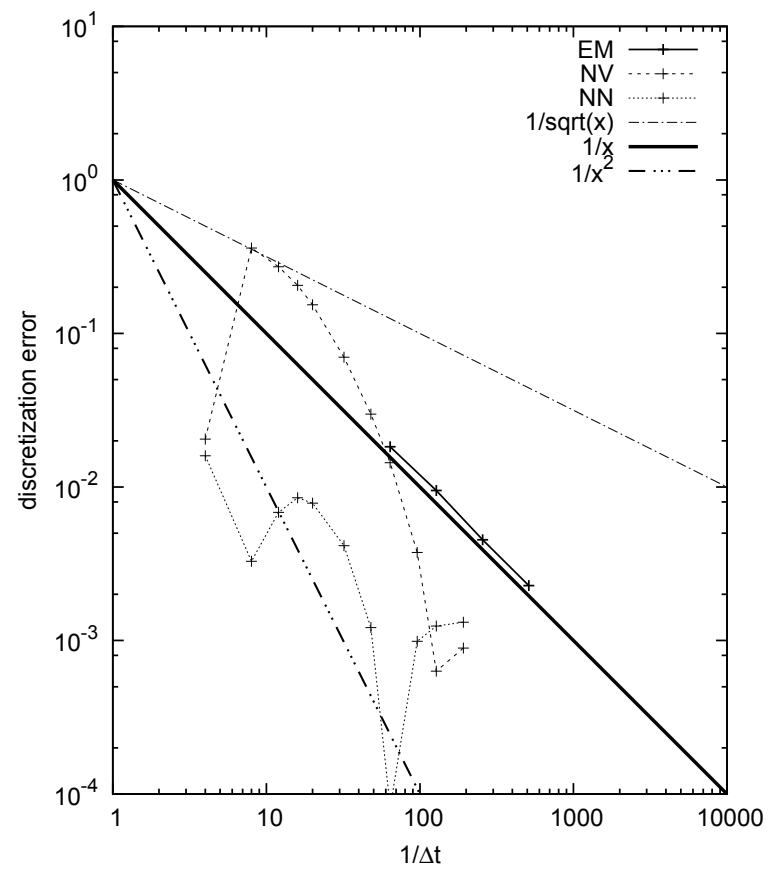

F. 5.7: (drift-2) $\quad x=1.0, g:$ call, $k=k_{n}$

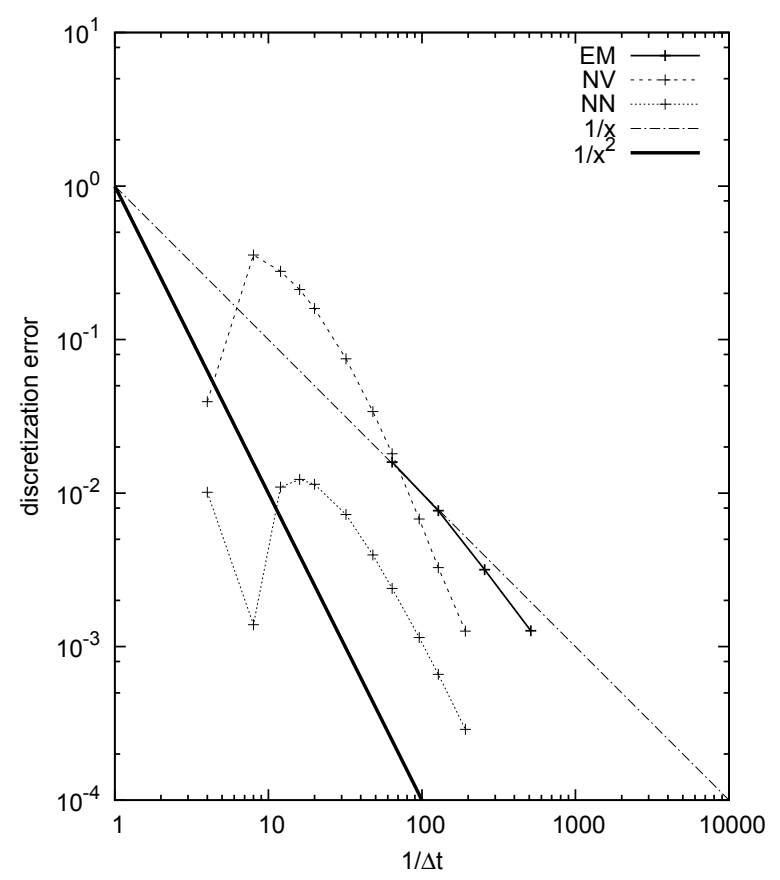

F. 5.8: $\left(\right.$ drift-2) $\quad x=1.0, g:$ call, $k=k_{s}$

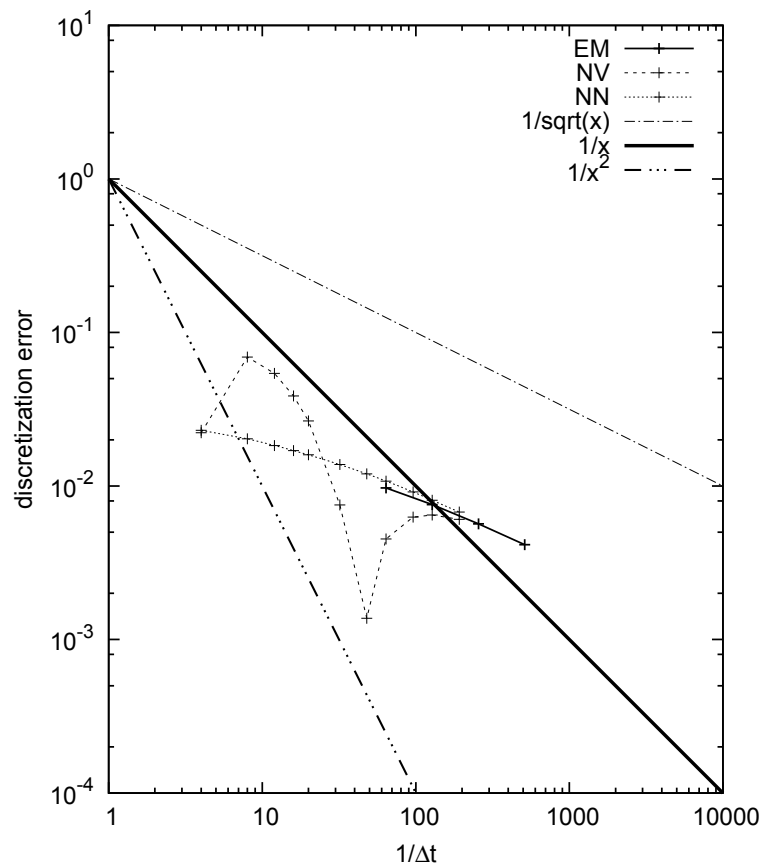

F. 5.9: (drift-2) $x=0.75, g:$ digital, $k=k_{n}$

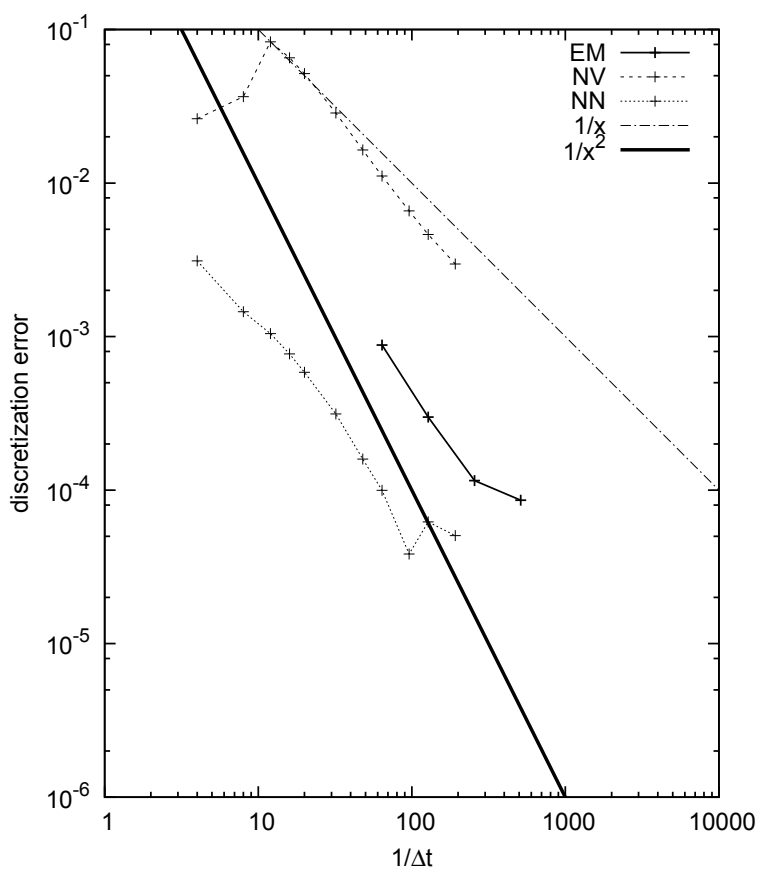

F. 5.10: (drift-2) $\quad x=0.75, g:$ digital, $k=k_{s}$ 


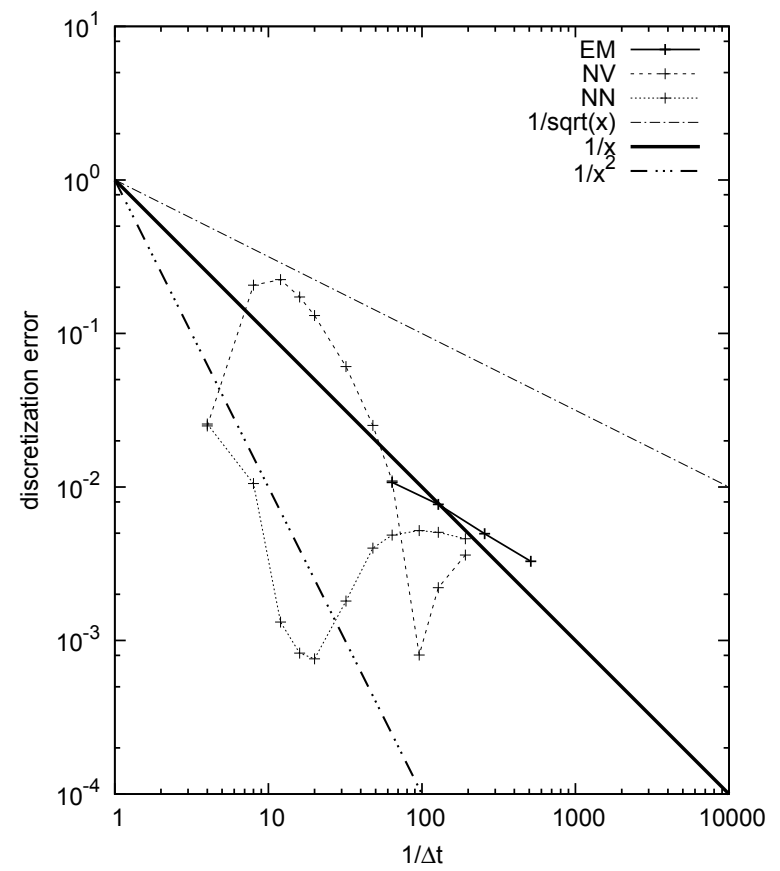

F. 5.11: (drift-2) $x=0.75, g:$ call, $k=k_{n}$

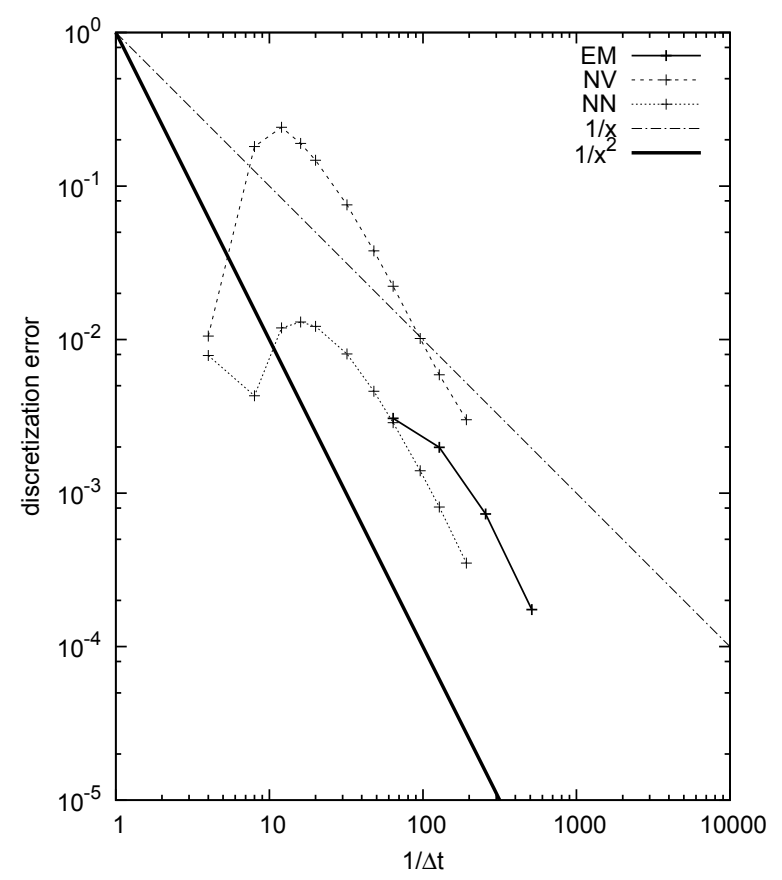

F. 5.12: (drift-2) $x=0.75, g:$ call, $k=k_{s}$

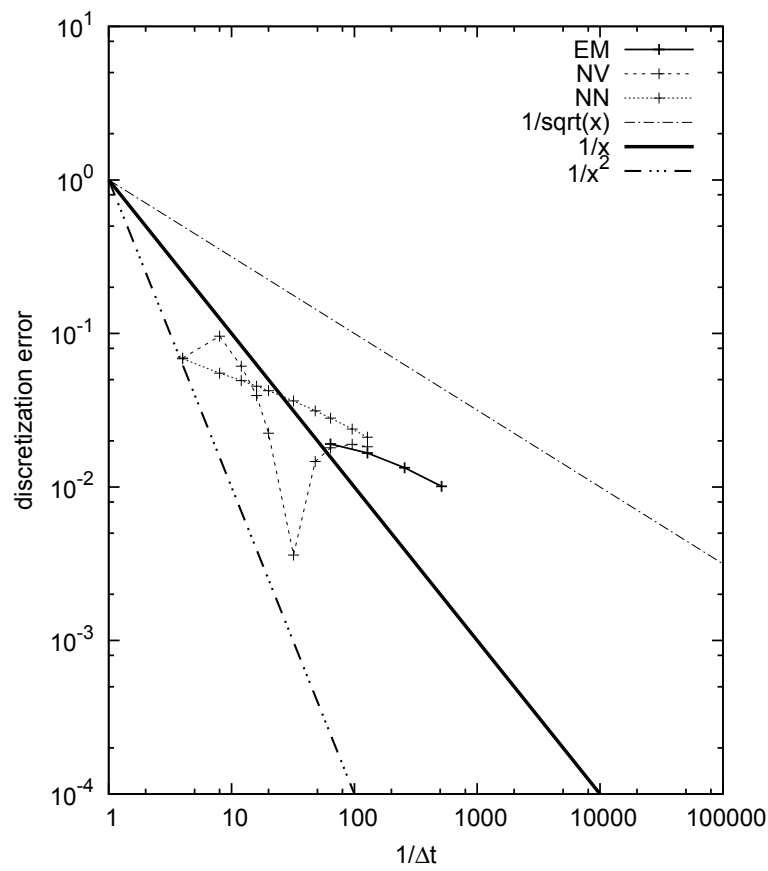

F. 5.13: (drift-2) $x=0.5, g:$ digital, $k=k_{n}$

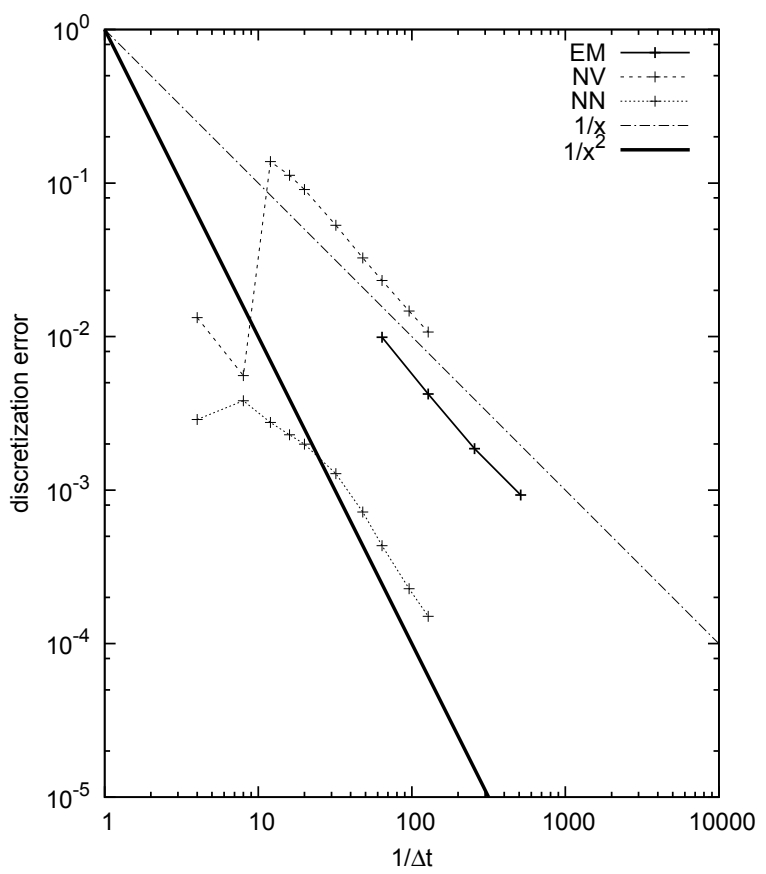

F. 5.14: (drift-2) $\quad x=0.5, g:$ digital, $k=k_{s}$ 


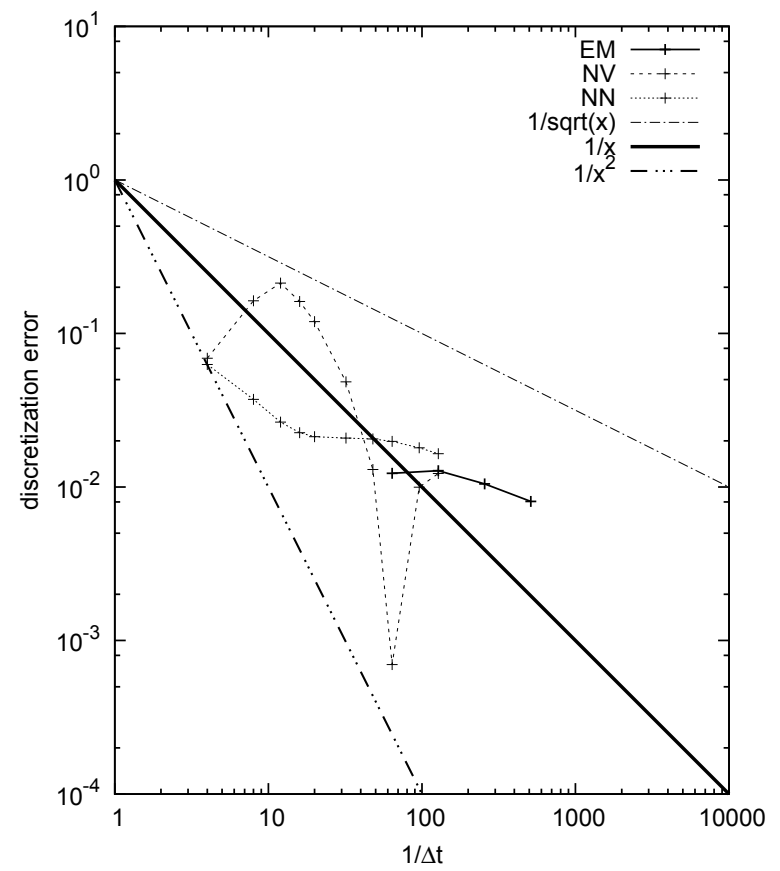

F. 5.15: (drift-2) $x=0.5, g:$ call, $k=k_{n}$

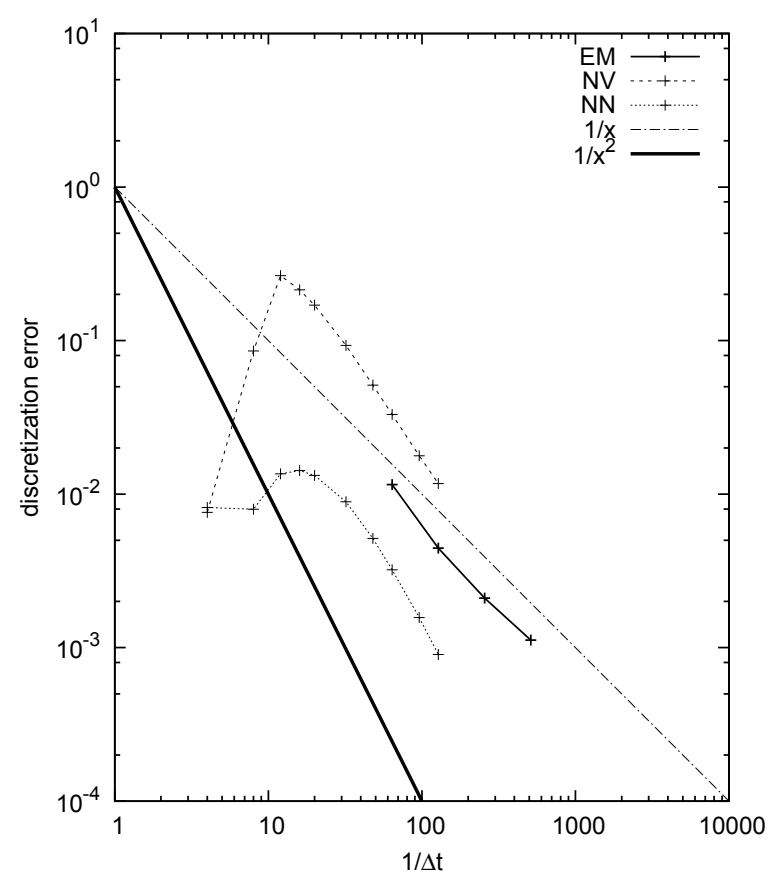

F. 5.16: (drift-2) $\quad x=0.5, g:$ call, $k=k_{s}$

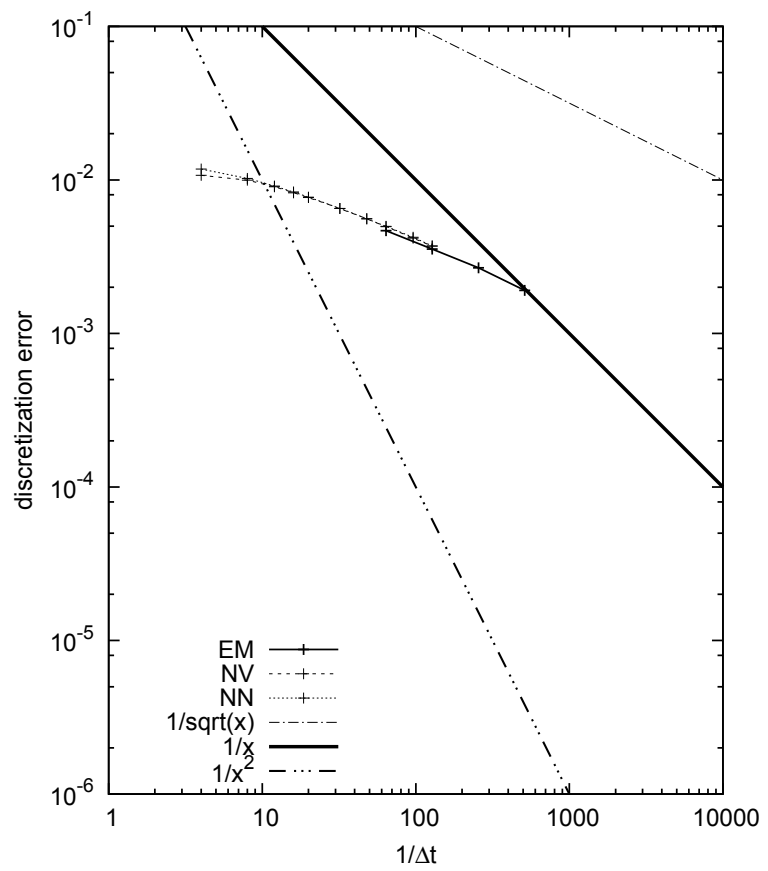

F. 5.17: (drift-3) $g$ : digital, $\quad k=k_{n}$

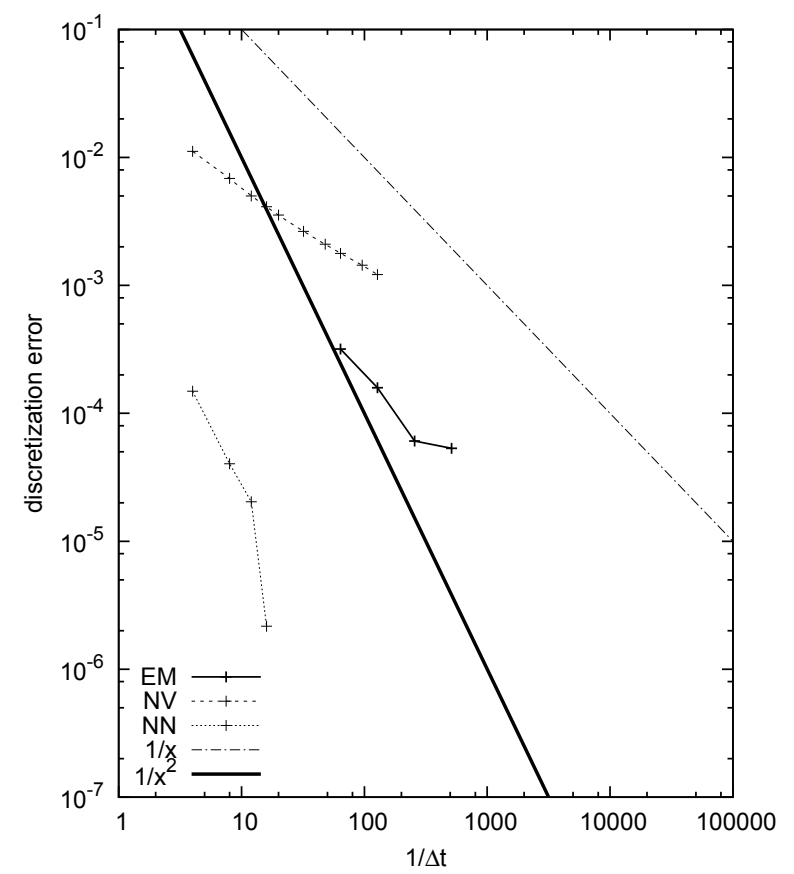

F. 5.18: (drift-3) $g:$ digital, $k: \operatorname{std}$ 


\section{Discussion}

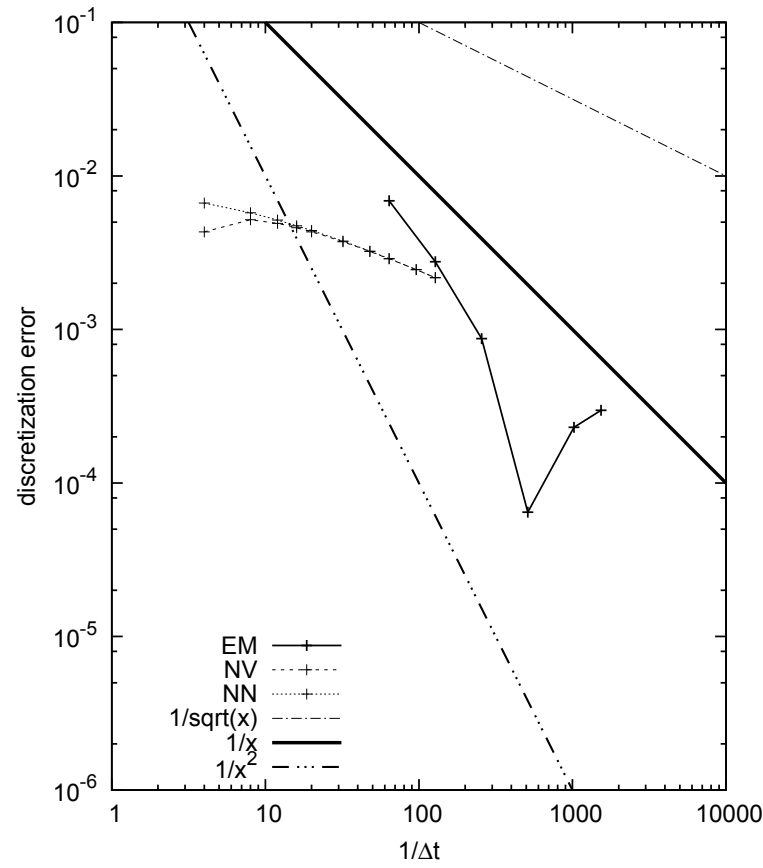

F. 5.19: (drift-3) $g:$ call, $\quad k=k_{n}$

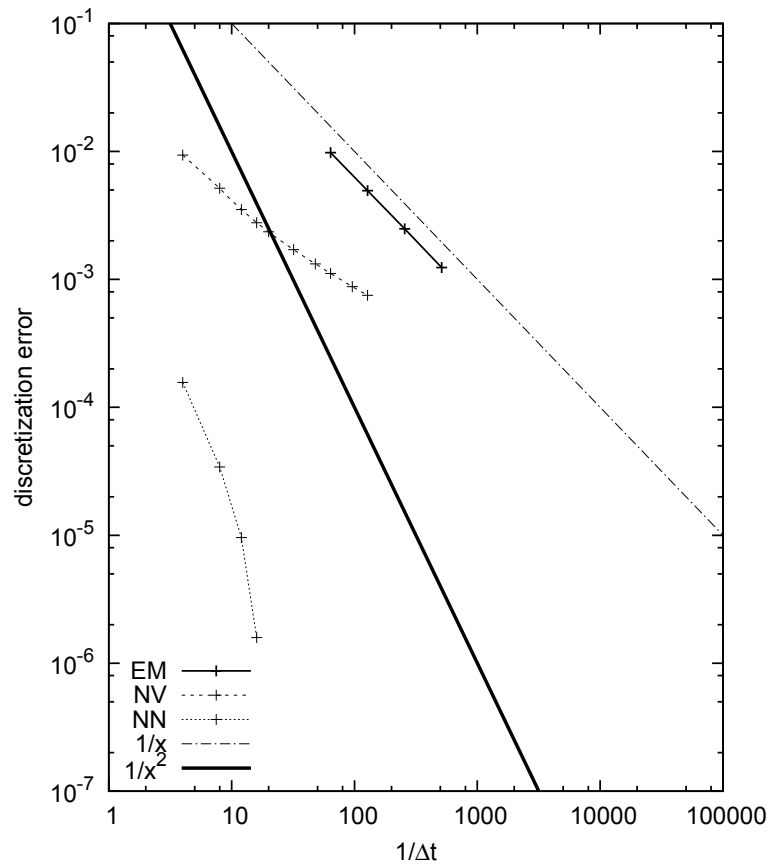

F. 5.20: (drift-3) $g$ : call, $k=k_{s}$
We observe the following facts from the graphs showing the discretization error in the previous section.

- The boundary condition makes consequences remarkably different from the European-type problems.

- The NN algorithm performs better than the other in most models.

- As long as using the naive killing function, we could not expect steeper slope than $-1 / 2$ for any algorithms.

- The absolute error of the NV and the NV algorithms show markedly differ depending on the situation. Also, the order of the NV algorithm could be less than that of the NN algorithm.

- As the initial value $x$ gets close to 0 , which means that the probability of hitting boundary increases, the effect of killing functions become clearer.

The following themes would be the next step in our research :

- Great contribution of killing functions is revealed in the numerical experiments. Hence, better approximation for the NV and the NN algorithms could be expected by improving killing functions in the sense of order of approximation.

- Since $\gamma$ plays an important role in approximation as shown in [7], the numerical experiments should be carried out with various values of $\gamma$.

- The range of the models should be extended to higher-dimensional SDEs, which means $d \geq 2$.

\section{Acknowledgments}

This research was partly supported by JSPS KAKENHI Grant-in-Aid for Young Scientists(B) 23730297 and Grant-in-Aid for Scientific Research(C) 22540115.

\section{References}

[1] V. Bally and D. Talay. The law of the Euler scheme for stochastic differential equations I. Convergence rate of the distribution function. Probability theory and related fields, 104:43-60, 1996.

[2] Philipp Drsek, Josef Teichmann, and Dejan Veluek. Cubature methods for stochastic (partial) differential equations in weighted spaces. Stochastic Partial Differential Equations: Analysis and Computations, 1(4):634-663, 2013. 
[3] Takehiro Fujiwara. Sixth order methods of Kusuoka approximation. Preprint Series of the Graduate School of Mathematical Sciences, The University of Tokyo: UTMS 2006-7, 2006.

[4] Emmanuel Gobet. Weak approximation of killed diffusion using euler schemes. Stochastic processes and their applications, 87(2):167-197, 2000.

[5] Benjamin Jourdain and Mohamed Sbai. High order discretization schemes for stochastic volatility models. Preprint: arXiv:0908.1926v3 [math.PR], pages 6-8, 2009.

[6] Peter E. Kloeden and Eckhard Platen. Numerical Solution of Stochastic Differential Equations. Springer Verlag, Berlin, 1999.

[7] Shigeo Kusuoka. Approximation of Expectation of Diffusion Process and Mathematical Finance. In T. Sunada, editor, Advanced Studies in Pure Mathematics, Proceedings of Final Taniguchi Symposium, Nara 1998, volume 31, pages 147-165, 2001.

[8] Shigeo Kusuoka. Approximation of Expectation of Diffusion Processes based on Lie Algebra and Malliavin Calculs. Advances in Mathematical Economics, 6:69-83, 2004.

[9] Terry Lyons and Nicolas Victoir. Cubature on Wiener Space. Proceedings of the Royal Society of London. Series A. Mathematical and Physical Sciences, 460:169-198, 2004.

[10] Mariko Ninomiya. Application of the Kusuoka approximation with a tree-based branching algorithm to the pricing of interest-rate derivatives under the HJM model. LMS Journal of Computation and Mathematics, 13:208-221, 2010.

[11] Mariko Ninomiya and Syoiti Ninomiya. A new higher-order weak approximation scheme forstochastic differential equations and the RungeKutta method. Finance and Stochastics, 13:415443, 2009. 10.1007/s00780-009-0101-4.

[12] Syoiti Ninomiya. A new simulation scheme of diffusion processes: Application of the Kusuoka approximation to Finance Problems. Mathematics and Computers in Simulation, 62/3-6:479-486, 2003.

[13] Syoiti Ninomiya. A partial sampling method applied to the Kusuoka approximation. Monte Carlo Methods and Applications, 9:27-38, 2003.

[14] Syoiti Ninomiya and Shu Tezuka. Toward real-time pricing of complex financial derivatives. Applied Mathematical Finance, 3:1-20, 1996.
[15] Syoiti Ninomiya and Nicolas Victoir. Weak Approximation of Stochastic Differential Equations and Application to Derivative Pricing. Applied Mathematical Finance, 15(2):107-121, 2008.

[16] Kojiro Oshima, Josef Teichmann, and Dejan Veluscek. A new extrapolation method for weak approximation schemes with applications. Preprint: arXiv:0911.4380v1 [math.PR], 2009.

[17] Denis Talay and Luciano Tubaro. Expansion of the global error for numerical schemes solving Stochastic Differential Equations. Stochastic Analysis and Applications, 8:483-509, 1990. 